\title{
Monte Carlo study of a self-avoiding polymerized membrane with negative bending rigidity
}

\author{
Shintaro Mori†§ and Shigeyuki Komura $\ddagger \|$ \\ $\dagger$ Department of Physics, Graduate School of Science, University of Tokyo, Hongo 7-3-1 \\ Tokyo 113, Japan \\ $\ddagger$ Department of Mechanical System Engineering, Kyushu Institute of Technology, Iizuka 820, \\ Japan
}

Received 26 April 1996, in final form 24 July 1996

\begin{abstract}
We study the possibility of a cancellation between the entropic bending rigidity due to self-avoiding interaction and negative bending rigidity. Results of Monte Carlo simulation show that a self-avoiding polymerized membrane with negative bending rigidity exhibits a phase transition from the usual floppy flat phase to a rigid folded flat (thin plate) phase. Even for flexible membranes, the complete cancellation does not occur. Based on these results, we discuss the problems in theoretical approaches to the phase transitions of polymerized membranes.
\end{abstract}

\section{Introduction}

There has been considerable interest in the understanding of statistical properties of polymerized (or tethered) membranes [1-3]. One of the surprising features is that the self-avoiding polymerized membrane is flat when it is embedded in three dimensional space [4-6]. The flat phase observed in the computer simulations was partly explained in terms of the crumpling transition induced by the implicit entropic bending rigidity due to the self-avoidance [7]. In fact, the restriction of the excluded volume interactions between the next-nearest-neighbour particles through the core potential inevitably induces the bending rigidity and prevents the membranes from bending freely. It is claimed in [7] that the previous simulations of the hard sphere model did not treat the pure self-avoiding effect and attributed the flatness to the model-dependent problems.

On the other hand, there is an interesting report insisting that polymerized membranes with self-avoiding interactions are always flat even for small diameters of hard spheres [5]. By reducing the size of the hard spheres, it is possible to remove the effect of the entropic bending rigidity from the next-nearest-neighbour core potential and one can study the pure self-avoiding effect. We also note a different computational method of enforcing self-avoidance in a model system by using a triangulated surface at which no vertex can cross a plane of a triangle created by any three neighbouring atoms $[8,9]$. There is a discrepancy between the results of these papers and we cannot draw definite conclusions. Here, we assume that the self-avoiding membrane is flat even with this model, although the crossovers are extremely slow [10].†

$\S$ E-mail address: mori@monet.phys.s.u-tokyo.ac.jp

|| E-mail address: komura@iizuka.isc.kyutech.ac.jp

$\dagger$ The results obtained in [8,9] and in [10] are different. As in [11], we rely on the latter one. 
According to the paper by Kantor and Kremer [11], the model of a polymerized membrane was described by a local Hamiltonian with repulsive interactions acting only between hard spheres whose degree of neighbourhood does not exceed a certain value $l$. They found that the hard sphere diameter $\sigma$ has a $l$ dependent critical value $\sigma_{\mathrm{c}}(l)$ at which the membrane undergoes a crumpling transition. Moreover, a scaling relation such that $\sigma_{\mathrm{c}}(l)^{3} \sim l^{-4}$ has been found. This indicates that the self-avoiding membrane becomes flat even for hard spheres of small diameter. Hence, the flatness is considered to be an inherent property of self-avoiding polymerized membranes. In addition, these authors proposed the following picture of the behaviour of the self-avoiding membrane. On a very short length scale, the self-avoiding interaction is irrelevant and the membrane is crumpled. Up to a certain length scale $l_{\text {crmp }}$, self-avoiding interactions produce an effective rigidity that suffices to keep the entire membrane flat. Within this picture, the crumpling transition is brought about by the effective rigidity. Beyond the scale $l_{\text {crmp }}$, the membrane is flat and the self-avoiding interaction no longer plays an essential role.

While it was established that the self-avoiding polymerized membrane is flat in computer simulations, there is still no satisfactory theory which explains this flatness. By applying a Gaussian variational technique to the generalized Edwards model, it was found that the membranes are crumpled above space dimension $d=4$ and the radius of gyration $R_{\mathrm{g}}$ scales with the linear membrane size $L$ as $R_{\mathrm{g}} \sim L^{v}$ where $v=4 / d$ [12-14]. This exponent gives a good estimate for the results of computer simulations in higher space dimensions $d \geqslant 4$ [15]. Their theory, however, can only treat the crumpled phase and does not tell anything about the crumpling transition induced by the entropic rigidity. In addition, one cannot answer why the Gaussian variational technique, instead of the Flory approximation [2] or the perturbative renormalization method [16], does work in the membrane case.

Recently, the phase transition of polymerized membranes with attractive interactions has been investigated by several people [7,17-21]. Extensive computer simulations have shown that there are two types of phase transition, depending on the flexibility of the membrane [19]. When the membrane is less flexible, it undergoes a first-order phase transition separating a high-temperature flat phase and a low-temperature collapsed phase. In the presence of an open boundary, the membrane exhibits a well defined sequence of folding transitions [17]. When the membrane is flexible, on the other hand, the transition becomes either continuous or weakly first order. By assuming that the transition is continuous, the exponent for the radius of gyration $v$ at the transition point is estimated as $v=0.85$.

Phenomenological aspects of these phase transitions can be described by the Landau model proposed by Abraham and Kardar [17, 20]. The above-explained different behaviours depending on the flexibility of the membrane are discussed in the following way [20,21].

An attractive interaction induces negative bending rigidity when the membrane is flat and cancellation can occur between this negative rigidity and the entropic rigidity due to the self-avoiding interactions. When the membrane is less flexible, the flat phase becomes unstable before complete cancellation occurs and the transition becomes discontinuous or first order. As for flexible membranes, the above cancellation can be complete and the crumpling transition might occur. In this continuous transition, an intermediate crumpled phase appears before the membrane becomes a collapsed phase. However, these theoretical approaches incorporate the effect of a self-avoiding interaction through an effective bending rigidity and only treat the instability of flat polymerized membranes without self-avoidance.

In this paper, we discuss the problem of cancellation between the entropic rigidity and the negative bending rigidity both by theoretical arguments and Monte Carlo simulation. We especially pay attention to the possibility of complete cancellation.

The outline of the present paper is as follows. In section 2, we use the generalized 
Edwards model to describe the flat phase of the membrane. We obtain the relation between the $\sigma_{\mathrm{c}}(l)$ and $l$ according to the scaling argument. In section 3, we explain the model used for the Monte Carlo simulation. The total Hamiltonian includes both self-avoiding interactions and local bending energy with negative bending rigidity. The flexibility of the membrane can be changed by using different diameters of the hard spheres. Section 4 contains the results of our simulation. Our study shows that there occurs a phase transition from the usual flat phase to another rigid flat phase which we call the 'folded flat phase'. An intermediate crumpled phase does not appear even for small hard spheres, which indicates that complete cancellation does not occur.

\section{Excluded volume effects in the flat phase}

In this section, we shall use the Gaussian variational approximation [12-14] in order to discuss the properties of a self-avoiding polymerized membrane being in the flat phase. So far the self-avoiding interaction has always been considered to be irrelevant when the membrane is flat [1]. This picture seems correct intuitively. However, if the self-avoidance is irrelevant for the flat membrane, then what makes the membrane flat? To answer this question, we use the generalized Edwards model for a flat self-avoiding polymerized membrane.

According to the discussion by Kantor and Kremer [11], the membrane is crumpled at short length scales. Hence, at this length scale, we can use the generalized Edwards model,

$\mathcal{H}[\boldsymbol{X}(s)]=\frac{1}{2} \int \mathrm{d}^{D} s \partial_{\alpha} X^{i} \partial_{\alpha} X^{i}+u \int \mathrm{d}^{D} s \int \mathrm{d}^{D} s^{\prime} \delta^{d}\left[X^{i}(s)-X^{i}\left(s^{\prime}\right)\right]$.

Here, $X^{i}\left(s^{\alpha}\right)(i=1, \ldots, d, \alpha=1, \ldots, D)$ describes the position of the membrane with internal coordinates $s^{\alpha} . u$ represents the strength of the excluded volume interaction. We assume that the interaction $\delta^{d}\left[X^{i}(s)-X^{i}\left(s^{\prime}\right)\right]$ is valid only when $\left|s-s^{\prime}\right|$ is smaller than some length scale $l_{\text {crmp. }}$. When $\left|s-s^{\prime}\right|$ is greater than $l_{\text {crmp }}$, the self-avoiding interaction becomes irrelevant.

In order to estimate the effect of the self-avoidance at short length scales, we employ the usual variational method with the quadratic Hamiltonian in Fourier space with wavenumber $k[12,14]$ :

$$
\mathcal{H}_{0}=\int \mathrm{d}^{D} k X^{i}(-\boldsymbol{k}) g(k) X^{i}(\boldsymbol{k}) .
$$

From the variational equation with respect to $g(k)$, it is possible to obtain the effective rigidity $\kappa_{\text {eff }}$ due to the self-avoidance of strength $u$ as

$$
\kappa_{\text {eff }}=\kappa_{\text {bare }}+c_{1} u \int \mathrm{d}^{D} s s^{4}\left\langle|\boldsymbol{X}(s)-\boldsymbol{X}(0)|^{2}\right\rangle^{-1-d / 2}
$$

where $\left\langle|\boldsymbol{X}(s)-\boldsymbol{X}(0)|^{2}\right\rangle$ is the two-point function averaged over the variational Hamiltonian equation (2.2) and $c_{1}$ is some constant. We have also added the constant bare bending rigidity $\kappa_{\text {bare }}$ for later convenience. We note that this equation is valid only in the crumpled part of the membrane, i.e. up to some length scale $l_{\text {crmp }}$. Beyond this length scale, the effect of the self-avoidance turns out to be irrelevant. Thus the length scale $l_{\text {crmp }}$ is determined by the condition that the effective bending rigidity becomes large such that the crumpling transition to the flat phase occurs. Therefore, the value of the effective rigidity equation (2.3) is almost fixed at the critical value $\kappa_{\mathrm{c}}$ of the crumpling transition of the membrane without self-avoidance. 
In [11], the range of the neighbourhood $l$ and the critical diameter of the hard sphere $\sigma_{\mathrm{c}}$ are determined by the condition at which the crumpling transition occurs. In this case, $l$ can be identified with $l_{\text {crmp }}$ and $u$ corresponds to $\sigma_{\mathrm{c}}^{d}$. Although the behaviour of $\left\langle|\boldsymbol{X}(s)-\boldsymbol{X}(0)|^{2}\right\rangle$ near the length scale $l_{\text {crmp }}$ is not clear, we simply assume here that $\left\langle|\boldsymbol{X}(s)-\boldsymbol{X}(0)|^{2}\right\rangle=c_{2} s^{2}$ with some constant $c_{2}$ since the membrane is almost flat near the length scale $l_{\text {crmp. }}$. Then the integral in equation (2.3) can be estimated to behave as

$$
\int \mathrm{d}^{2} s s^{4}\left(c_{2} s^{2}\right)^{-1-d / 2} \sim l_{\text {crmp }}^{4-d}
$$

for $d<4$ and we obtain the following relation,

$$
\kappa_{\mathrm{c}}=\kappa_{\text {bare }}+c_{3} u l_{\mathrm{crmp}}^{4-d}
$$

where $c_{3}$ is a positive constant. If we put $\kappa_{\text {bare }}=0$, equation (2.5) corresponds to the relation obtained by Kantor and Kremer†. In fact, what they have obtained is $u l^{4} \sim 1$ at $d=3$.

Next we consider the effect of the negative bending rigidity $\left(\kappa_{\text {bare }}<0\right)$. If we assume that the system exhibits the crumpling transition as a result of the complete cancellation between the effective entropic rigidity and the negative rigidity, the length scale $l_{\text {crmp }}$ should be the order of membrane size $L$. Therefore, the critical value of the negative rigidity $\kappa_{\text {bare }}^{0}$ at which the crumpling transition occurs is determined by

$$
\kappa_{\text {bare }}^{0}=\kappa_{\mathrm{c}}-c_{3} u L^{4-d} .
$$

From this equation, we see that $\kappa_{\text {bare }}^{0}$ depends both on $u$ and $L$, as long as the crumpling transition occurs. In the following section, we examine this possibility by Monte Carlo simulation.

\section{Model and simulation method}

In this section we describe the model for the polymerized membrane used in the computer simulations and summarize the computational procedure. The model membrane being embedded in three-dimensional space consists of hard spheres connected with flexible bonds. As shown in figure 1, hard spheres are connected in a two-dimensional triangular array and the polymerized membrane of finite size forms a hexagonal cluster. There are $N=\left(3 L^{2}+1\right) / 4$ spheres in such a membrane, where $L$ is the number of spheres on the diameter. For the present simulation, we employ a commonly used tethering interaction, i.e. the spheres interact with their nearest neighbours through the potential

$$
V_{\mathrm{NN}}(r)= \begin{cases}0 & \sigma<r<\sqrt{3} \sigma \\ \infty & \text { otherwise. }\end{cases}
$$

This potential consists of a hard core repulsive region which prevents the neighbouring spheres from occupying the same space and a finite region where the spheres can move freely. In addition to this neighbouring potential, self-avoiding interactions have been included by restricting the distance between all the pairs of the spheres to be greater than $\sigma$. In order to change the flexibility of the membrane, we allow $\sigma$ to take several values $(<1.0)$ although it is usually set equal to 1.0. In this case the model has the drawback that the self-intersection is not necessarily prohibited. The membrane can be flexible and we will study such a case.

$\dagger$ The discrepancy between theoretical and numerical results is considered to be from the simple assumption for the two-point function. If we assume that $\left\langle|\boldsymbol{X}(s)-\boldsymbol{X}(0)|^{2}\right\rangle=c_{2} s^{v}$ with $v<2.0$, the correspondence becomes better. 


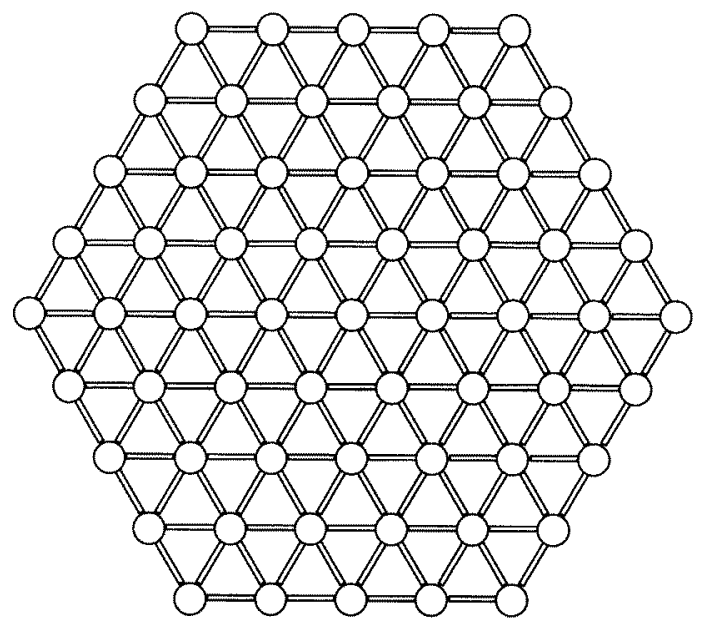

Figure 1. A model of the polymerized membrane with linear size $L=9$.

We also take into account the bending energy in the model by introducing the repulsive spring between next-nearest-neighbour spheres in the network [4]

$$
\mathcal{H}_{\mathrm{b}}=-\frac{\kappa^{\prime}}{2} \sum_{\mathrm{NNN}}\left|\boldsymbol{X}(\sigma)-\boldsymbol{X}\left(\sigma^{\prime}\right)\right|^{2} .
$$

In this case, the summation is taken over all next-nearest neighbours in the network. So far, the bending rigidity parameter $\kappa^{\prime}$ has been set as positive numbers, which implies that the bending energy forces the membranes to become flat. In contrast, we consider her an anti-ferromagnetic-like interaction, namely, the rigidity parameter $\kappa^{\prime}$ takes negative values. If this interaction cancels the entropic bending energy completely, one might expect the occurrence of a crumpling transition separating the flat phase and the crumpled phase. At sufficiently large values of $\left|\kappa^{\prime}\right|$, the membranes are expected to be in the folded state. We have used the standard Monte Carlo procedure (Metropolis algorithm) to investigate the thermodynamic properties of self-avoiding polymerized membranes with negative bending rigidity. The total Hamiltonian is the sum of equations (3.1) and (3.2) with the self-avoiding interactions.

The random displacement of step size $s \leqslant 0.2$ is attempted on a randomly selected sphere in the network and this procedure is repeated until the system reaches the thermal equilibrium state. Let $T$ denote the absolute temperature and $k_{\mathrm{B}}$ the Boltzmann constant. The attempted movement is always accepted if the energy $E_{\mathrm{t}}$ of the trial movement does not exceed the original energy $E_{0}$. If $E_{\mathrm{t}}$ exceeds $E_{0}$, the move is accepted with the probability $\exp \left[\left(E_{0}-E_{\mathrm{t}}\right) / k_{\mathrm{B}} T\right]$. The sequence of this procedure depends on the dimensionless parameter $\kappa=\left|\kappa^{\prime}\right| / k_{\mathrm{B}} T$ which is the measure for the temperature scale. Since the tethering potential equation (3.1) has zero or infinite energy, the corresponding probability is either 1 (accepted) or 0 (rejected). For the bending energy equation (3.2), one has to evaluate the probability $\exp \left[\left(E_{0}-E_{\mathrm{t}}\right) / k_{\mathrm{B}} T\right]$ for each value of $\kappa$. Due to the restriction of selfavoidance, the distances between all the pairs in the network must be checked during the simulation. Thus the procedure takes quite a long time for the system to reach equilibrium configurations.

The relaxation time $\tau$ is determined by the slowest mode corresponding to large-scale motions. For phantom membranes, $\tau$ is given by the Rouse relaxation time $\tau \approx N / s^{2}$. 
It takes a much longer time for self-avoiding membranes to reach the equilibrium state, which is one of the difficulties in carrying out the simulation. In order to know the typical relaxation time, we have traced the behaviour of the eigenvalues of the inertia tensor whose sum is equal to the square of the radius of gyration. Although each eigenvalue has different relaxation time, the dynamics of the radius of gyration is dominated by the slowest time. Through the analysis of auto-correlation functions, we found that the relaxation time is the order of a few hundred Rouse times for $L=11,13$ and 17. For all of the runs, we have collected at least 100 samples and in most cases 500 samples separated in time by such a relaxation time.

\section{Results}

We have calculated the statistical properties of polymerized membranes over the range $0.0 \leqslant \kappa \leqslant 9.0\left(-9.0 \leqslant \kappa^{\prime} / k_{\mathrm{B}} T \leqslant 0\right)$. We have first investigated the membranes of sizes $L=11,13$ and 17 with the conventional diameter $\sigma=1.0$. For the membranes of size $L=17$, unfortunately, equilibrium states were not realized for $\kappa>7.0$ in our computer system. To investigate the features of the membrane conformations, we have calculated the inertia tensor $I_{\alpha \beta}$ defined by

$$
I_{\alpha \beta}=\frac{1}{N} \sum_{i} X_{i \alpha} X_{i \beta}-\frac{1}{N^{2}} \sum_{i, j} X_{i \alpha} X_{j \beta}
$$

where the summation is over the spheres for each configuration and the Greek subscripts $\alpha$ and $\beta$ denote the components of three-dimensional coordinates of the sphere. The summation of three eigenvalues of the inertia tensor denoted by $\lambda_{1}, \lambda_{2}$ and $\lambda_{3}\left(\lambda_{1} \leqslant \lambda_{2} \leqslant \lambda_{3}\right)$ is equal to the squared radius of gyration $R_{\mathrm{g}}^{2}$ defined by

$$
R_{\mathrm{g}}^{2}=\frac{1}{2 N^{2}} \sum_{i, j}\left|\boldsymbol{X}_{i}-\boldsymbol{X}_{j}\right|^{2}
$$

In the flat phase, the eigenvalues scale with the linear membrane size $L$ as $\lambda_{1} \sim L^{2 v_{1}}$, and $\lambda-2 \sim \lambda_{3} \sim L^{2 v_{3}}$ where $v_{3} \simeq 1.0$. Hence the radius of gyration which is dominated by the largest eigenvalue $\lambda_{3}$ scales as $R_{\mathrm{g}}^{2} \sim L^{2}$. $v_{1}$ corresponds to the roughness exponent and some computer simulations estimated its value as $0.5 \sim 0.8[4,6]$.

In contrast to the flat phase, the membranes are isotropic in the crumpled or compact phase. In this case, all the three eigenvalues and the squared radius of gyration scale with the same exponent $v_{1}=v_{2}=v_{3}=v<1.0$. We have looked at each eigenvalue to find the scaling behaviour of the membranes because the used system sizes are rather small. Figure 2 shows three eigenvalues of the inertia tensor as a function of $\kappa$ for the membrane size $L=13$. It can be seen that all three eigenvalues decrease as $\kappa$ increases. For small $\kappa$, it is expected that the self-avoiding interaction dominates the negative bending forces. Thus the membranes should be asymptotically flat. As $\kappa$ increases, the flat phase becomes unstable and the membranes are expected to be driven into folded states. for large $\kappa$, the two largest eigenvalues $\lambda_{2}$ and $\lambda_{3}$ converges to the same value, while the smallest eigenvalue $\lambda_{1}$ approaches a constant value.

To investigate the qualitative measure of anisotropic configurations of membranes, we have calculated the asphericity $A$ defined as the ratio of the largest to the smallest eigenvalue such that

$$
A(\kappa, L)=\left\langle\lambda_{1} / \lambda_{3}\right\rangle
$$




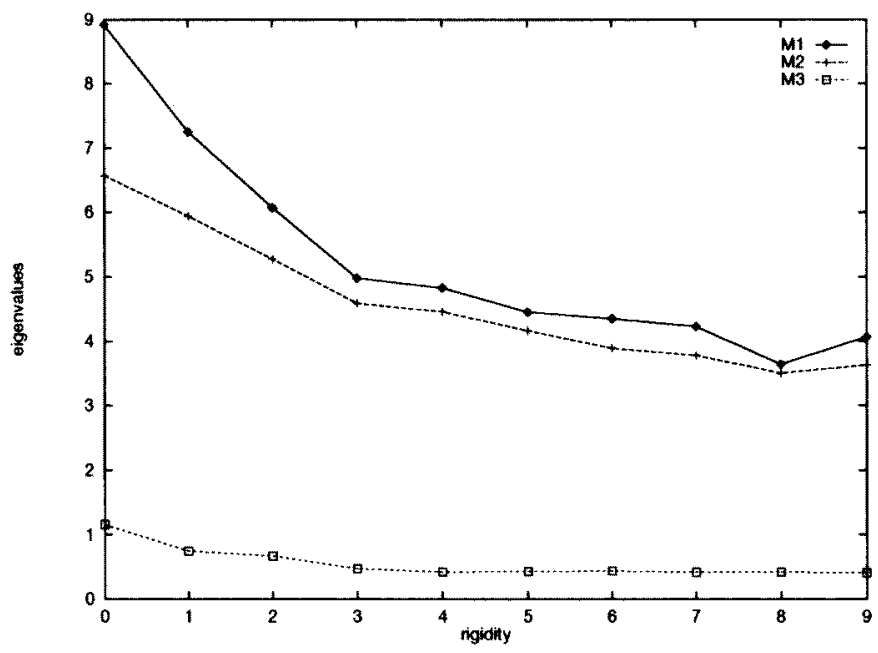

Figure 2. Three eigenvalues of inertia tensor as a function of rigidity for the size $L=13$.

Hereafter $\langle\cdots\rangle$ denotes the configurational average. Figure 3 depicts $A(\kappa, L)$ for $L=11,13$ and 17. One can see that the asphericity is less than 0.2 for all $\kappa$. For fixed $L$, the asphericity decreases to a minimal value and then increases as $\kappa$ is increased. For all values of $\kappa, A$ decreases with increasing $L$. In the limit of $L \rightarrow \infty$, we expect that $A$ tends to zero independent of $\kappa$. These results suggest that the membranes are anisotropic for all values of $\kappa$ and the isotropically crumpled or collapsed phase will not appear.

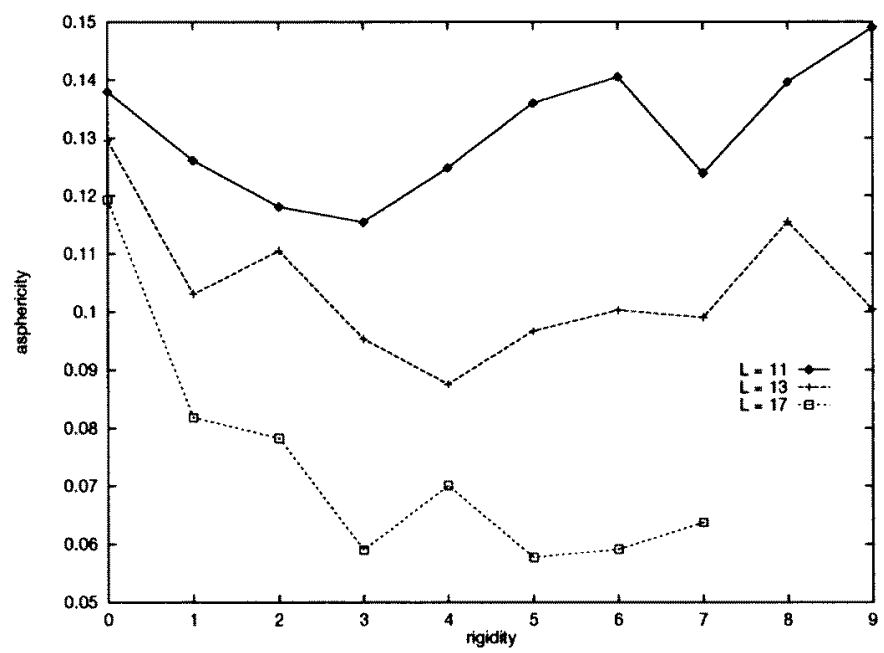

Figure 3. Asphericity $A$ as a function of rigidity parameter $\kappa$ for $L=11,13$ and 17 .

We have also attempted to determine the exponent $s v_{1}$ and $v_{3}$ for each different value of $\kappa$. The results are summarized in table 1 . We find that $\nu_{3}$ is almost equal to 1.0 for all $\kappa$ values within numerical uncertainty. On the other hand, $\nu_{1}$ approaches zero as $\kappa$ increases, indicating that the smallest eigenvalue is independent of $L$ and approaches a universal non-zero constant at large $\kappa$. We have also checked that the relation $v_{2}=v_{3}$ holds. 
Table 1. The exponents $v_{3}$ and $v_{1}$ obtained from data $L=11,13$ and 17 .

\begin{tabular}{lll}
\hline$\kappa$ & $\nu_{3}$ & $v_{1}$ \\
\hline 0.0 & $0.94 \pm 0.01$ & $0.45 \pm 0.09$ \\
1.0 & $0.95 \pm 0.01$ & $0.45 \pm 0.12$ \\
2.0 & $0.91 \pm 0.01$ & $0.41 \pm 0.11$ \\
3.0 & $0.97 \pm 0.02$ & $0.19 \pm 0.05$ \\
4.0 & $0.94 \pm 0.03$ & $0.30 \pm 0.07$ \\
5.0 & $1.03 \pm 0.01$ & $0.05 \pm 0.03$ \\
6.0 & $0.99 \pm 0.01$ & $0.01 \pm 0.01$ \\
7.0 & $0.84 \pm 0.01$ & $0.07 \pm 0.02$ \\
\hline
\end{tabular}

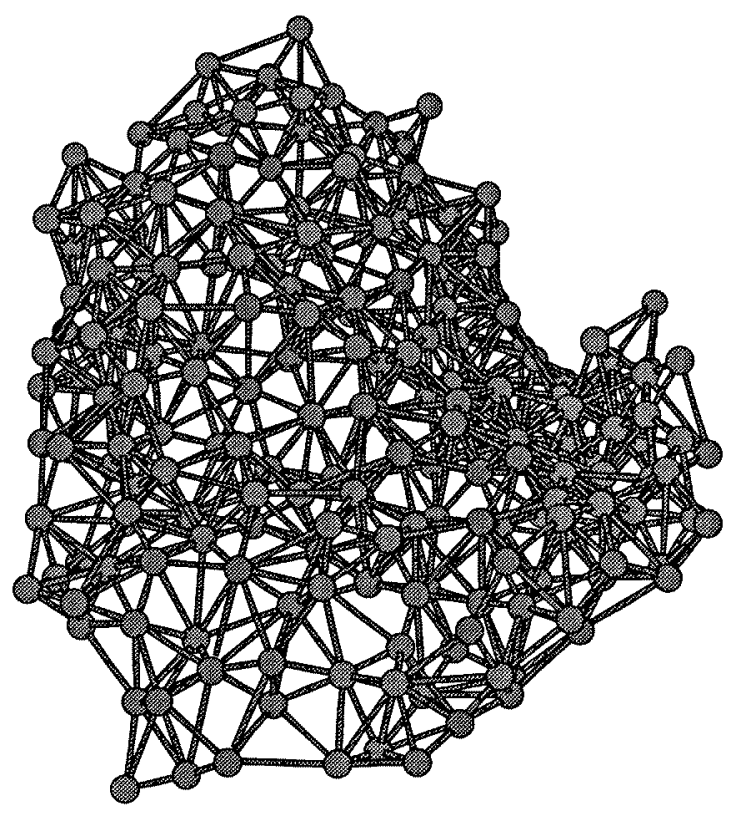

Figure 4. The equilibrium configuration of the hexagonal membrane with $L=17$ for $\kappa=1.0$. This picture corresponds to the flat phase.

In figure 4, a typical equilibrium configuration of membrane size $L=17$ for $\kappa=1.0$ is depicted. This picture corresponds to the flat phase. Figure 5 represents the case for $\kappa=7.0$. Now the membrane is anisotropically folded and forms a hexagonal thin plate with a finite width. From these observations, fixing $\sigma=1.0$, membranes with negative bending rigidity exhibit a transition from the flat phase to the folded rigid flat states by increasing $\kappa$. Since an intermediate crumpled phase does not appear, the crumpling transition does not occur.

In view of the above results, we could not find any evidence for the existence of the intermediate crumpled phase. In order to investigate the more flexible case, we have studied membranes with smaller spheres, i.e. $\sigma=0.5$ and 0.3. We have determined the exponents $\nu_{3}$ and $\nu_{1}$ from the system sizes $L=11,13$ and 17. Table 2 summarizes the results for several values of $\kappa$. Again, $\nu_{2}$ is close to $\nu_{3}$. Similar to the case of $\sigma=1.0$, there is no isotropic intermediate crumpled phase even if the size of hard spheres is reduced.

In our simulation, we have also obtained other thermodynamic functions such as internal energy or specific heat. In contrast to the situation of phantom membranes with bending 


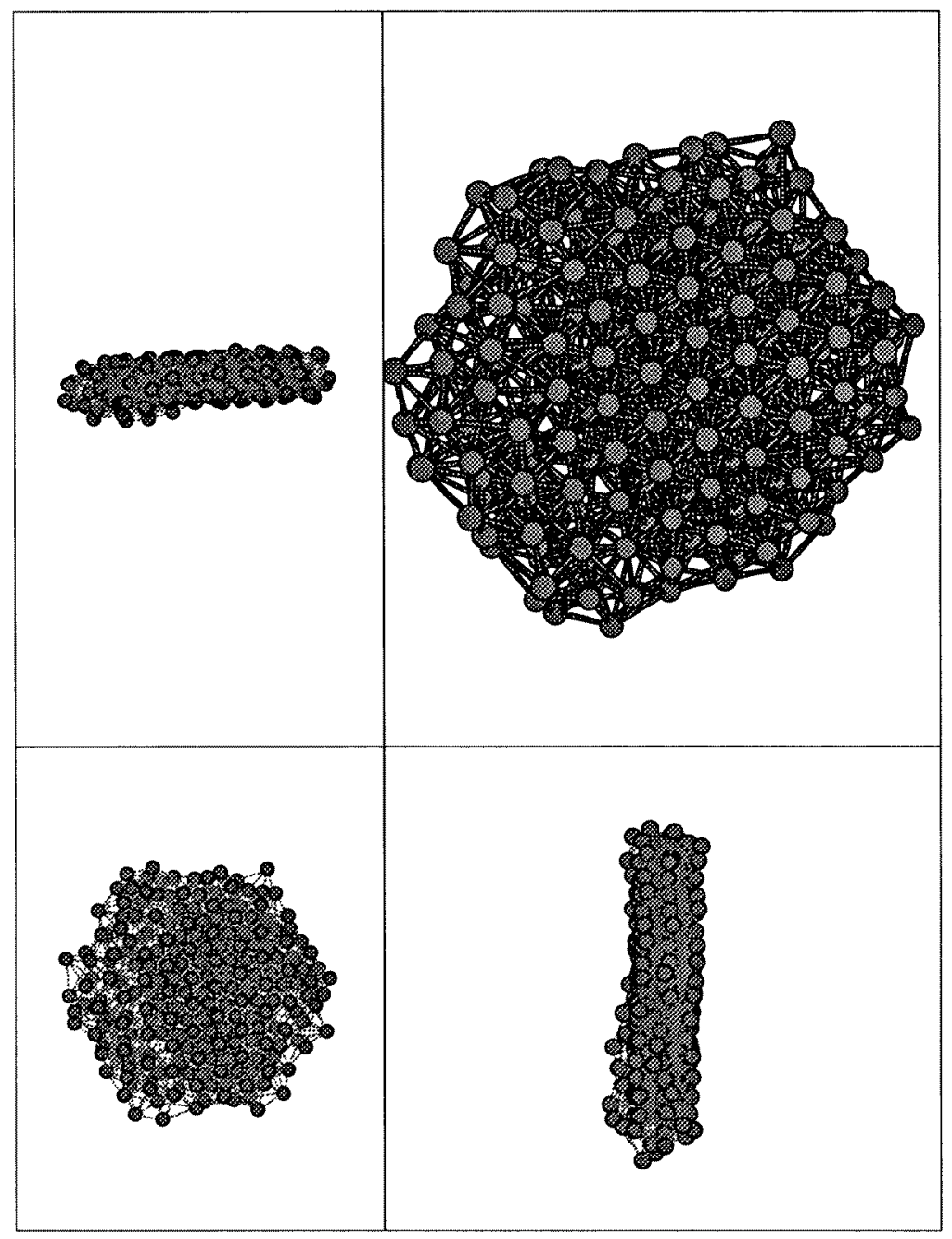

Figure 5. Typical configuration of $L=17$ membrane with $\kappa=7.0$. The perspectives except for the upper right picture are along the directions of the principal axes of the three eigenvalues.

energy [2], we only found a vague peak for the case of $\sigma=1.0$ and no clear singularity in the smaller sphere cases. Much more investigations are required in order to discuss the nature of the phase transitions.

\section{Discussion}

In the theoretical part of this paper, we have used the generalized Edwards model to describe the flat phase of the polymerized membrane. Within the picture by Kantor and Kremer, we have obtained the scaling relation. In addition, we have discussed the possibility of the cancellation between the entropic rigidity and the negative bending rigidity, which might cause the crumpling transition. 
Table 2. Scaling exponents of maximum and minimum eigenvalues for diameter $\sigma=0.5$ and 0.3 .

\begin{tabular}{llllll}
\hline & \multicolumn{2}{c}{$\sigma=0.5$} & & \multicolumn{2}{c}{$\sigma=0.3$} \\
\cline { 2 - 3 } \cline { 5 - 6 }$\kappa$ & $v_{3}$ & $v_{1}$ & & $v_{3}$ & $v_{1}$ \\
\hline 1.0 & $0.87 \pm 0.02$ & $0.47 \pm 0.12$ & & $0.82 \pm 0.01$ & $0.38 \pm 0.11$ \\
2.0 & $0.74 \pm 0.02$ & $0.46 \pm 0.09$ & & $0.73 \pm 0.03$ & $0.30 \pm 0.07$ \\
3.0 & $0.87 \pm 0.03$ & $0.30 \pm 0.11$ & & $0.80 \pm 0.01$ & $0.24 \pm 0.08$ \\
4.0 & $0.90 \pm 0.01$ & $0.12 \pm 0.03$ & & $0.78 \pm 0.01$ & $0.18 \pm 0.04$ \\
5.0 & $0.90 \pm 0.02$ & $0.08 \pm 0.02$ & & $0.78 \pm 0.02$ & $0.21 \pm 0.02$ \\
6.0 & $0.89 \pm 0.01$ & $0.08 \pm 0.01$ & & $0.81 \pm 0.01$ & $0.23 \pm 0.04$ \\
\hline
\end{tabular}

However, the results of the Monte Carlo simulation showed that, instead of the crumpling transition, the membrane exhibits a phase transition from the flat phase to the rigid folded flat phase. An intermediate crumpled phase does not appear. We have also investigated the membranes with more local flexibility consisting of smaller hard spheres $(\sigma>0.3)$. Even in this case, the crumpled phase has not been observed within our simulation. From these results, we conclude that the phase transition from the flat phase to the rigid folded flat phase occurs before the negative rigidity cancels the entropic rigidity completely. At this point, the flat phase of the self-avoiding membrane is different from that of the phantom membrane, which shows the crumpling transition.

We thing that complete cancellation occurs only in the very flexible membrane with an attractive interaction and hence the continuous transition is expected. Theoretical approaches to describe the transition have incorporated the effect of the self-avoiding interaction through the bending rigidity and treated only the stability of the flat phase of the phantom membrane [20]. In such a treatment, both negative rigidity and an attractive interaction can cause the crumpling transition. Our computer simulation, however, has made it clear that we cannot incorporate the effect of the self-avoiding interaction through the effective bending rigidity even if the membrane is flexible. Therefore, one must be careful about the conclusion which is based on the identification of the flat self-avoiding membrane with the flat phantom membrane.

Up to now, theoretical arguments concerning the stability of the flat phase have been limited to phantom membranes. As far as the self-avoidance is concerned, we can only discuss the existence region of the crumpled phase. These points are the most difficult aspects in studying the polymerized membranes theoretically. That is, if one predicts some instability in the flat phantom membrane, such phenomenon may not occur in the selfavoiding membrane.

Finally, we comment that the phase transition under consideration is different from the one discussed by David, Francesco and Guitter [22,23]. What they studied is the phase transition of the folding square lattice with negative bending rigidity and they showed that there is a first-order transition separating the flat phase and the folded compact state. Since hard cores are of finite size in our simulation, the membrane takes the folded flat state rather than the folded compact state. However, since our simulations have been performed on rather small system sizes, we could not accurately determine the properties of the transition. In addition, the size of the hard spheres should be smaller in order to conclude whether or not complete cancellation occurs for a very flexible membrane. We think that more extensive simulations should be performed in the future. 


\section{Acknowledgments}

The authors thank K Kawanishi for collaborations and useful discussions. One of the authors (SM) thanks M Wadati for useful discussions and critical reading of the manuscript. He also thanks J Nishimura for useful discussions that motivated this work.

\section{References}

[1] Nelson D R, Piran T and Weinberg S (eds) 1989 Statistical mechanics of membranes and surfaces Proc. of the 5th Jerusalem Winter School (Singapore: World Scientific)

[2] Kantor Y, Kardar M and Nelson D R 1986 Phys. Rev. Lett. 57 791; 1987 Phys. Rev. A 353056

[3] Kantor Y and Nelson D R 1987 Phys. Rev. Lett. 58 2774; 1987 Phys. Rev. A 364020

[4] Plischke M and Boal D 1988 Phys. Rev. A 384943

[5] Boal D, Levinson E, Liu D and Plischke M 1989 Phys. Rev. A 403292

[6] Abraham F F, Rudge W and Plischke M 1989 Phys. Rev. Lett. 621757

[7] Abraham F F and Nelson D R 1990 J. Physique 51 2653; 1990 Science 249393

[8] Baumgärtner A 1991 J. Physique I 11549

[9] Baumgärtner A and Renz W 1992 Europhys. Lett. 17381

[10] Kroll D M and Gompper G 1993 J. Physique I 31131

[11] Kantor Y and Kremer K 1993 Phys. Rev. E 482490

[12] Guitter E and Palmeri J 1992 Phys. Rev. A 45734

[13] Le Doussal P 1992 J. Phys. A: Math. Gen. 25 L469

[14] Goulian M 1991 J. Physique II 11327

[15] Grest G S 1991 J. Physique I 11695

[16] David F and Weise K 1996 Phys. Rev. Lett. 764564 and references therein

[17] Abraham F F and Kardar M 1991 Science 252393

[18] Liu D and Plischke M 1992 Phys. Rev. A 457139

[19] Grest G S and Petsche I B 1994 Phys. Rev. E 50 R1737

[20] Mori S and Wadati M 1995 Phys. Lett. 201A 61

[21] Mori S and Kajinaga Y 1996 Phys. Rev. E 53124

[22] David F and Guitter E 1988 Europhys. Lett. 5709

[23] Di Francesco P and Guitter E 1994 Phys. Rev. E 504418 